\title{
An Error-Resilient and Tunable Distributed Indexing Scheme for Wireless Data Broadcast
}

\author{
Jianliang $\mathrm{Xu}^{1}$, Wang-Chien Lee ${ }^{2}$, Xueyan Tang ${ }^{3}$, Qing Gao ${ }^{1}$, Shanping $\mathrm{Li}^{1 *}$ \\ ${ }^{1}$ Hong Kong Baptist University, Kowloon Tong, Hong Kong \\ ${ }^{2}$ Penn State University, University Park, PA \\ ${ }^{3}$ Nanyang Technological University, Singapore
}

\begin{abstract}
Access efficiency and energy conservation are two critical performance concerns in a wireless data broadcast system. We propose in this paper a novel parameterized index called the exponential index that has a linear yet distributed structure for wireless data broadcast. Based on two tuning knobs, index base and chunk size, the exponential index can be tuned to optimize the access latency with the tuning time bounded by a given limit, and vice versa. The client access algorithm for the exponential index under unreliable broadcast is described. A performance analysis of the exponential index is provided. Extensive ns-2 based simulation experiments are conducted to evaluate the performance under various link error probabilities. Simulation results show that the exponential index substantially outperforms the state-of-the-art indexes. In particular, it is more resilient to link errors and achieves more performance advantages from index caching. The results also demonstrate its great flexibility in trading access latency with tuning time.
\end{abstract}

Index Terms: Index structure, data broadcast, energy conservation, mobile computing

\section{Introduction}

Wireless data broadcast has received a lot of attention from industries and academia in recent years. It has been available as commercial products for many years (e.g., StarBand [20] and Hughes Network [21]). In particular, the recent announcement of the smart personal objects technology (SPOT) by Microsoft [16] further highlights the industrial interest in and feasibility of utilizing broadcast for wireless data services. With a continuous broadcast

\footnotetext{
${ }^{*}$ The work was done when he was a Visiting Scholar at HKBU. He is with College of Computer Science, Zhejiang University, Hangzhou 310027, China.
} 
network (called DirectBand Network) using FM radio subcarrier frequencies, SPOT-based devices (e.g., PDAs and watches) can continuously receive timely information such as stock quotes, airline schedules, local news, weather, and traffic information.

Access efficiency and energy conservation are two main performance issues for the clients in a wireless data broadcast system. Access efficiency concerns how fast a request is satisfied, and energy conservation concerns how to reduce a mobile client's energy consumption when it accesses the data of interest. While access efficiency is a constantly tackled issue in most system and database research, energy conservation is very critical due to the limited battery capacity on mobile clients [25]. To facilitate energy conservation, a mobile device typically supports two operation modes: active mode and doze mode. The device normally operates in the active mode; it can switch to the doze mode to save energy when the system becomes idle. For example, a typical wireless PC card, ORiNOCO, consumes $60 \mathrm{~mW}$ during the doze mode and 805-1,400 $\mathrm{mW}$ during the active mode [25]. In the literature, two performance metrics, namely access latency and tuning time, have been used to measure access efficiency and energy conservation, respectively $[6,9,10]$ :

- Access latency: The time elapsed between the moment when a query is issued and the moment when it is responded.

- Tuning time: The amount of time a mobile client stays active to receive the requested data.

To retrieve a data item from wireless data broadcast, the mobile client has to continuously monitor the broadcast until the data arrives. This will consume a lot of energy since the client has to remain active during its waiting time. A solution to this problem is air indexing. The basic idea is to include some index information about the arrival times of data items on the broadcast channel. By accessing the index, the mobile client is able to predict the arrival of its desired data. Thus, it can stay in the doze mode during waiting time and tune into the broadcast channel only when the data item of interest arrives. Several traditional disk-based indexing techniques such as $\mathrm{B}^{+}$-tree have been extended for air indexing $[4,10,19]$. However, existing designs are mostly based on centralized tree structures, which are not performance 
efficient for the sequential-access broadcast media. Specifically, to start an index search, the client needs to wait until it reaches the root of the next broadcast search tree; also in case of link errors during index search, the client has to restart the search from the next broadcast root. ${ }^{1}$ To reduce such access delay, multiple replicated indexes are usually interleaved with data broadcast. Nevertheless, the air indexing solution has the drawback of lengthening the broadcast cycle due to the indexing information. In other words, there is a trade-off between access latency and tuning time.

Different application scenarios may require different performance trade-offs. For example, in an office environment, users may favor a short latency since they can easily recharge the batteries; while in the airport, users may prefer to conserve energy at the cost of a longer latency. As such, we need a tunable air indexing scheme to accommodate different requirements. A good air indexing scheme should be able to facilitate latency bounded tuning and tuning-time bounded tuning. In general, a shorter tuning time is expected when a longer latency can be tolerated, and vice versa. However, most of the existing indexing techniques are not flexible in the sense that the trade-off between tuning time and access latency is not adjustable based on application specific requirements.

In this paper, we propose a novel parameterized index called the exponential index. The proposed exponential index is very efficient because it naturally facilitates the index replication by sharing links in different search trees and thus minimizes storage overhead. Moreover, it has a linear yet distributed structure which suits the sequential-access broadcast environment very well. It not only allows searching to start at any index segment but also makes recovering an index search from a link error quickly. Based on its two tuning knobs, the exponential index can also be easily adjusted to optimize the access latency (or tuning time) with the tuning time (or access latency) bounded by a given limit. ${ }^{2}$

Wireless transmission is error-prone. Data might be corrupted or lost due to many factors like signal interference, etc. The client access algorithm for the exponential index

\footnotetext{
${ }^{1}$ This problem can be alleviated a little by enhancing the index with some auxiliary data structure [23].

${ }^{2}$ Note that it is not necessary for the whole system to employ a single tuning parameter. A wireless network typically consists of a large number of cells. The users in the same cell are likely to have similar performance favors. Thus, each cell can independently employ its own tuning parameter and apply the proposed work.
} 
under unreliable broadcast is described. We also provide a performance analysis of the exponential index in terms of the access latency and tuning time under unreliable wireless broadcast environments. Extensive experiments are conducted to compare the exponential index with two state-of-the-art air indexing schemes, i.e., the distributed tree [10] and the flexible index [9], under various link error probabilities. Simulation results show that the proposed exponential index substantially outperforms the existing indexing schemes. In particular, it is more resilient to link errors and achieves more performance advantages from index caching. The results demonstrate its great flexibility in trading access latency with tuning time.

The rest of this paper is organized as follows. Section 2 gives the background for indexing data on broadcast channels and reviews the related work. In Section 3, we introduce the proposed exponential index for clustered broadcast and explain how to tune different tradeoffs between tuning time and access latency. We compare the proposed index with the existing indexes in Section 4. Section 5 extends the exponential index to non-clustered broadcast and index caching. Finally, the paper is concluded in Section 6.

\section{Background}

\subsection{Preliminaries}

Consider a data dissemination system that periodically broadcasts a collection of data items (e.g., stock quotes) to mobile clients through a wireless broadcast channel. Each data item is a tuple of attribute values and can be identified by a key value. Similar to [10], the smallest access unit of a broadcast is referred to as a bucket, which physically consists of a fixed number of packets - the basic unit of message transfer in the network. Each bucket is identified by a sequentially increased id number. We distinguish between index buckets that hold the index and possibly some data if space permits, and data buckets that hold the

data (one or more items) only. A sequence of multiplexed index buckets and data buckets constitute a bcast, in which each data item appears at least once (see Figure 1). Bcasts can be classified as flat broadcast where each item appears exactly once, and skewed broadcast 


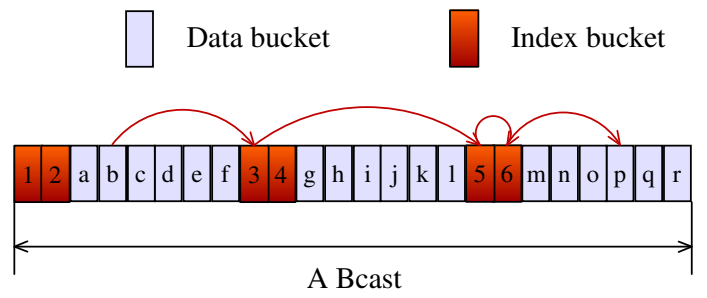

Figure 1: Data Organization on a Wireless Broadcast Channel

where some items may appear more than once. A bcast is repeatedly broadcast on the wireless channel.

To facilitate a data search via an air index, each data bucket includes an offset to the beginning of the next index bucket. Taking Figure 1 as an example, the general access protocol for retrieving data involves the following phases:

- Initial probe: The client tunes into the broadcast channel at bucket $b$ and determines when the next index bucket 3 is broadcast. The client tunes in again at bucket 3 .

- Index search: The client selectively accesses a number of index buckets (i.e., index buckets 3,5 , and 6) to find out when to get the desired data held in bucket $p$.

- Data retrieval: When bucket $p$ arrives, the client downloads it and retrieves the desired data. Thus, the access latency is 19 buckets; as the client needs to stay active for buckets $b, 3,5,6$, and $p$ only, the tuning time is 5 buckets.

There are two basic data organizations with respect to an attribute within a bcast: clustered broadcast and non-clustered broadcast. A sequence of data items are clustered if all the data items with the same value of the attribute appear consecutively; otherwise, they are non-clustered. Clustered broadcast corresponds to flat broadcast with respect to the primary attribute, whereas non-clustered broadcast corresponds to flat broadcast with respect to a secondary attribute or skewed broadcast [10]. Without loss of generality, data items in clustered broadcast can be arranged in ascending order of the attribute values. For non-clustered broadcast, a bcast can be partitioned into a number of segments called metasegments, each of which holds a sequence of items with non-descending (or non-ascending) values of the attribute [10]. Thus, when we look at each individual meta-segment, the data 
items are clustered on that attribute and the indexing techniques developed for clustered broadcast are still applicable to a meta-segment. Therefore, we mainly focus on clustered broadcast when we describe the proposed index in Section 3 and extend the technique to non-clustered broadcast in Section 5.

\subsection{Related Work}

Several disk-based indexing techniques have been extended for air indexing. Imielinski et al. re-designed the $\mathrm{B}^{+}$-tree such that the leaf nodes store the arrival times of the data items [10]. A distributed indexing method was proposed to efficiently replicate and distribute the index tree in a bcast. Chen et al. and Shivakumar et al. considered unbalanced tree structures to optimize energy consumption for non-uniform data access $[4,19]$. These structures minimize the average index search cost by reducing the number of index searches for hot data at the expense of spending more on cold data. Tan and Yu discussed data and index organization under skewed broadcast [24]. Hashing and signature methods have also been suggested for wireless broadcast that supports equality queries $[7,9]$. Hu et al. showed that the signature method is particularly attractive for multi-attribute indexing [6]. However, none of these techniques is flexible in adjusting access latency and tuning time. Moreover, as they are extended from disk-based environments, which support random access, they are not natural for broadcast environments, where only sequential access is allowed, and hence, tedious adaptation is needed.

A flexible indexing method was proposed in [9]. The flexible index first sorts the data items in ascending (or descending) order of the search key values and then divides them into $p$ segments. The first bucket in each data segment contains a control index, which is a binary index mapping a given key to the segment containing the key, and a local index, which is an $m$-entry index mapping a given key to the bucket containing the key within the current segment. By tuning the parameters of $p$ and $m$, mobile clients can achieve either a good tuning time or a good access latency. However, [9] does not make it clear how flexibility can be measured. As we shall see in Section 4, the flexibility of this indexing method is quite limited. 


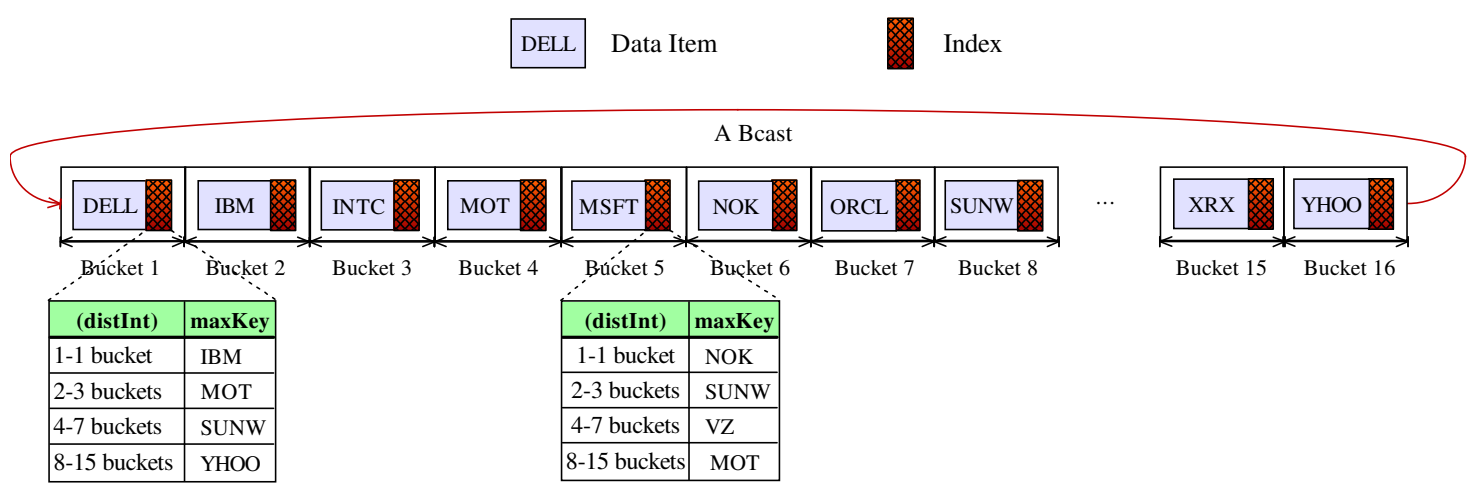

Figure 2: A Simple Exponential Index

While all the aforementioned work assumed an error-free broadcast environment, Tan and Ooi [23] investigated air indexing techniques for unreliable data broadcast. They enhanced the distributed tree index and the flexible index to efficiently deal with link errors. In this paper, we generalize our previously proposed exponential index [27] to an unreliable broadcast environment where link errors may occur. The proposed exponential index differs from the flexible index in at least three aspects: 1) instead of binary spaced indexing, the exponential index allows indexing spaces to be exponentially partitioned at any base value; 2) the exponential index intelligently exploits the available bucket space for indexing, whereas the flexible index blindly incurs overhead; and 3) the exponential index allows the current bcast to index into the next bcast to support an efficient search, but the flexible index indexes the data within the current bcast only.

Other related work investigated different aspects of broadcast, including data scheduling [5, 15], semantic broadcast [12], broadcast of location-dependent data [28], consistency management $[13,17]$, and cache management $[14,18,26]$. There also exist studies on designing error correction codes to improve data transmission reliability [8]. Complementary to these studies, we propose error-resilient data indexing methods to facilitate data accesses even if the broadcast is not reliable.

\section{The Exponential Index}

This section presents a new air indexing method called the exponential index. We focus on clustered broadcast in this section and shall extend the proposed index to non-clustered 
broadcast in Section 5. We first illustrate the basic idea of the exponential index by an example and then generalize it with two tunable parameters: index base and chunk size (to be defined later). Next, we analyze the performance of the generalized exponential index. Finally, we show how to adjust the trade-off between tuning time and access latency for the exponential index.

\subsection{A Motivating Example}

Consider a server that periodically broadcasts stock information (e.g., stock ticks, prices, trading volumes, etc). Suppose the server maintains 16 stock items that are arranged in a bcast in ascending order of their identifiers.

For simplicity, each bucket is assumed to accommodate only one stock item and some index information. ${ }^{3}$ As shown in Figure 2, a bcast consists of 16 buckets. Each bucket contains a data part and an index table. The index table consists of four entries (rows). Each entry indexes a segment of buckets in the form of a tuple $\{$ distInt, maxKey\}, where distInt specifies the distance range of the buckets from the current bucket (measured in the unit of buckets), and maxKey is the maximum key value of these buckets. The sizes of the segments grow exponentially. The first entry describes a single bucket segment (i.e., the next bucket), and for each $i>1$, the $i$ th entry describes the segment of buckets that are $2^{i-1}$ to $2^{i}-1$ away (i.e., $2^{i-1}$ buckets). Note that the distInt values need not be maintained in the index table since they can be inferred from their entry ids. The key range of the buckets indexed by the $i$ th entry is given by the maxKey values of the $(i-1)$ th and $i$ th entries.

Suppose that a client issues a query for item "NOK" right before item "DELL" (i.e., bucket 1) is broadcast. The client tunes into the broadcast channel and first retrieves the index table in bucket 1 (i.e., the left index table in Figure 2). Since "NOK" falls between the second maxKey "MOT" and the third maxKey "SUNW," the target item must lie in the buckets that are 4 to 7 away. The client then stays in the doze mode until bucket 5 is broadcast and examines the item in bucket 5. As the target item cannot be found in bucket 5 , the client further checks the index table in bucket 5 (i.e., the right index table in

\footnotetext{
${ }^{3}$ As mentioned, a bucket may accommodate one or more data items (i.e., stock items here), depending on the bucket capacity.
} 
Figure 2). Since "NOK" matches the first maxKey, the target item must be in the next bucket. Therefore, the client completes the query by accessing bucket 6 . The total tuning time for the query is 3 buckets (i.e., buckets 1, 5, and 6). Similarly, if a client wants to access item "SUNW" right before bucket 1 is broadcast, it can get the desired data by searching buckets 1, 5, 7, and 8. As we shall show in Section 3.3, the worst tuning time for this exponential index is $\left\lceil\log _{2}(N-1)+1\right\rceil$ buckets when the data broadcast is error-free, where $N$ is the total number of buckets in a bcast. In our example, where $N=16$, the worst tuning time is 5 buckets.

As mentioned, wireless data broadcast is unreliable. A bucket might be corrupted during broadcasting. We can handle such link errors easily with the exponential index. Again, using Figure 2 as an example, a client searches for "NOK" from the first bucket. If the broadcast is error-free, the client accesses buckets 1, 5, and 6, as discussed above. However, if bucket 1 is corrupted, the client immediately restarts the search from the next bucket (i.e., bucket 2). Thus, the client accesses bucket 1 (corrupted), and buckets 2 and 6 to get the desired data. If both buckets 1 and 2 are corrupted, the client restarts the search from bucket 3 and accesses buckets 5 and 6 . Hence, there is only a small performance penalty. This is indeed an advantage over the centralized tree index, where, if the root is corrupted, the client has to wait until the root is next broadcast before restarting the search, thus causing a significant access delay.

We can observe several nice properties of the exponential index from this simple example:

- The index has a linear yet distributed structure. Hence, it immediately enables an index search from the next index bucket (i.e., the next bucket with an index table), thereby saving access latency. The index bucket where a search starts represents the root of a search tree for the indexed data on the air.

- The index is naturally replicated in such a way that an index link is shared by different search trees, i.e., two index searches traversing through different search trees (i.e., starting with different root buckets) may use the same index links in the searching processes. Thus, the storage overhead of the index is minimized. 


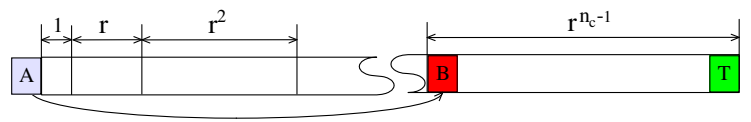

Figure 3: Illustration of Exponential Indexing Space

- The worst tuning time is the logarithm of the bcast length under error-free broadcast.

- The index can recover an index search from a link error quickly thanks to its distributed structure.

In addition, the next section shows that the indexing overhead can be controlled by adjusting the index structure (via an exponential base) and the number of index buckets.

\subsection{The Generalized Exponential Index}

In the above example, the sizes of the indexed segments exponentially increase by a base of 2 (hereafter referred to as the index base). To generalize the exponential index, the index base can be set to any value $r \geq 1$. Specifically, as shown in Figure 3, the $i$ th entry in the index table describes the maximum key value of a segment of $r^{i-1}$ buckets (i.e., the buckets that are $\left\lfloor\sum_{j=1}^{i-2} r^{j}+1\right\rfloor=\left\lfloor\frac{r^{i-1}-1}{r-1}+1\right\rfloor$ to $\left\lfloor\sum_{j=1}^{i-1} r^{j}\right\rfloor=\left\lfloor\frac{r^{i}-1}{r-1}\right\rfloor$ away).

Since the exponential index maintains an index table in each bucket, the bucket capacity to accommodate data items is reduced, thereby increasing the bcast length. To reduce such indexing overhead, we group $I$ buckets into a data chunk and build the exponential index on a per-chunk basis (i.e., including one index table in each chunk). This decreases the number of index tables in a bcast and the number of entries in each index table. However, the price to pay for per-chunk indexing is that an average of $\frac{I-1}{2}$ buckets need to be searched to locate a data item within a data chunk.

To remedy this, we propose, in a data chunk, to construct a plain index for all buckets, where an index entry is used to describe the maximum key value for each bucket. With the plain index, if we do not consider link errors, the intra-chunk tuning time is either 1 (for the first bucket in the chunk) or 2 buckets (for the other buckets). In this way, the index table for each data chunk is split into two parts: a global index for the other data chunks and a local index for the $I-1$ buckets within the local chunk. Figure 4 shows an example of the 


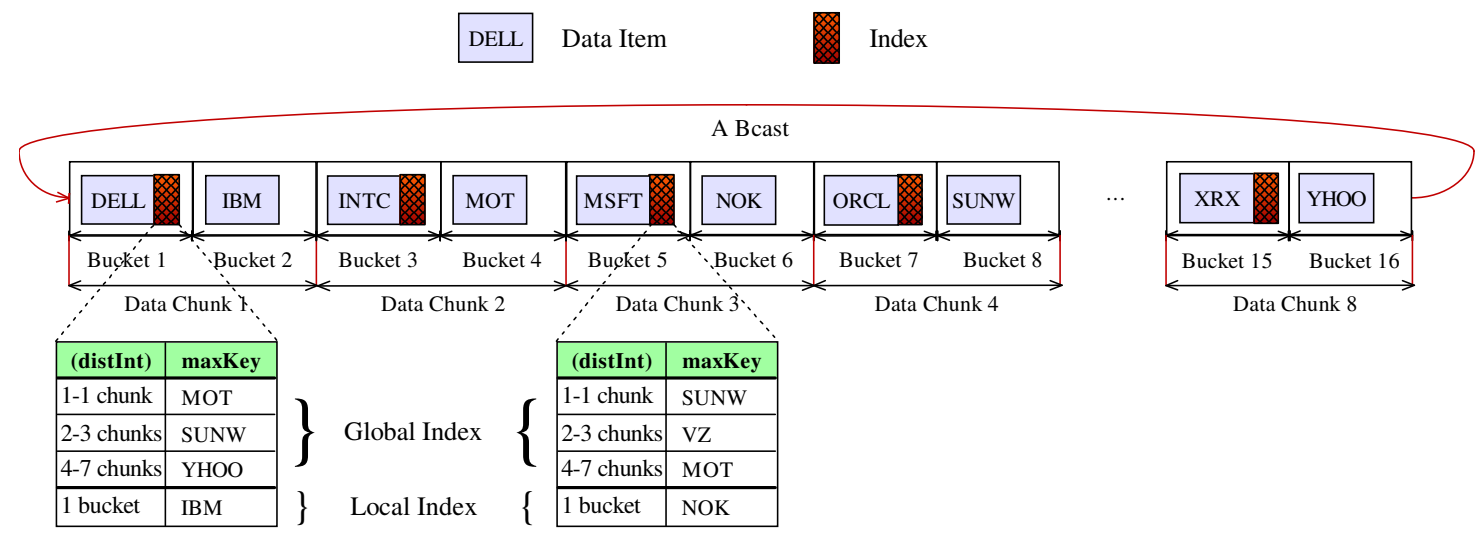

Figure 4: The Generalized Exponential Index $(r=2, I=2)$

generalized exponential index, where the index base $r$ is set at 2 and the chunk size $I$ is set at 2 .

We now describe the client access protocol under unreliable data broadcast. Assume that each data bucket includes an offset to the next index bucket (i.e., the first bucket of the next chunk). The client access protocol follows the same three phases described in Section 2.1. We discuss the access protocol for the proposed exponential index using an example (see Algorithm 1 for a formal description). Again, suppose that the client makes a query for item "NOK" right before the bucket containing item "DELL" is broadcast. Since the requested item is not in the current bucket, the client checks the local index. Because "NOK" is larger than the maximum key value "IBM" in the local index, the client proceeds to check the global index. In the global index, "NOK" lies in the key range specified by the second entry, hence the client goes into the doze mode and waits for the second next index bucket (i.e., bucket 5). In the index table of bucket 5, "NOK" falls in the key range specified by the first local index entry. Therefore, the client accesses the next bucket (i.e., bucket 6 ) to complete the query. The total tuning time is 3 buckets. As another example for the same index search, now suppose bucket 1 is corrupted. The client continues to access the next bucket 2 to find out the index bucket 3. From there, it proceeds to search bucket 5 and accesses bucket 6 to retrieve the desired data. If bucket 6 is also corrupted, the client waits for one bcast to download bucket 6 in the next broadcast cycle.

There are two tuning knobs for the generalized exponential index: index base $r$ and 


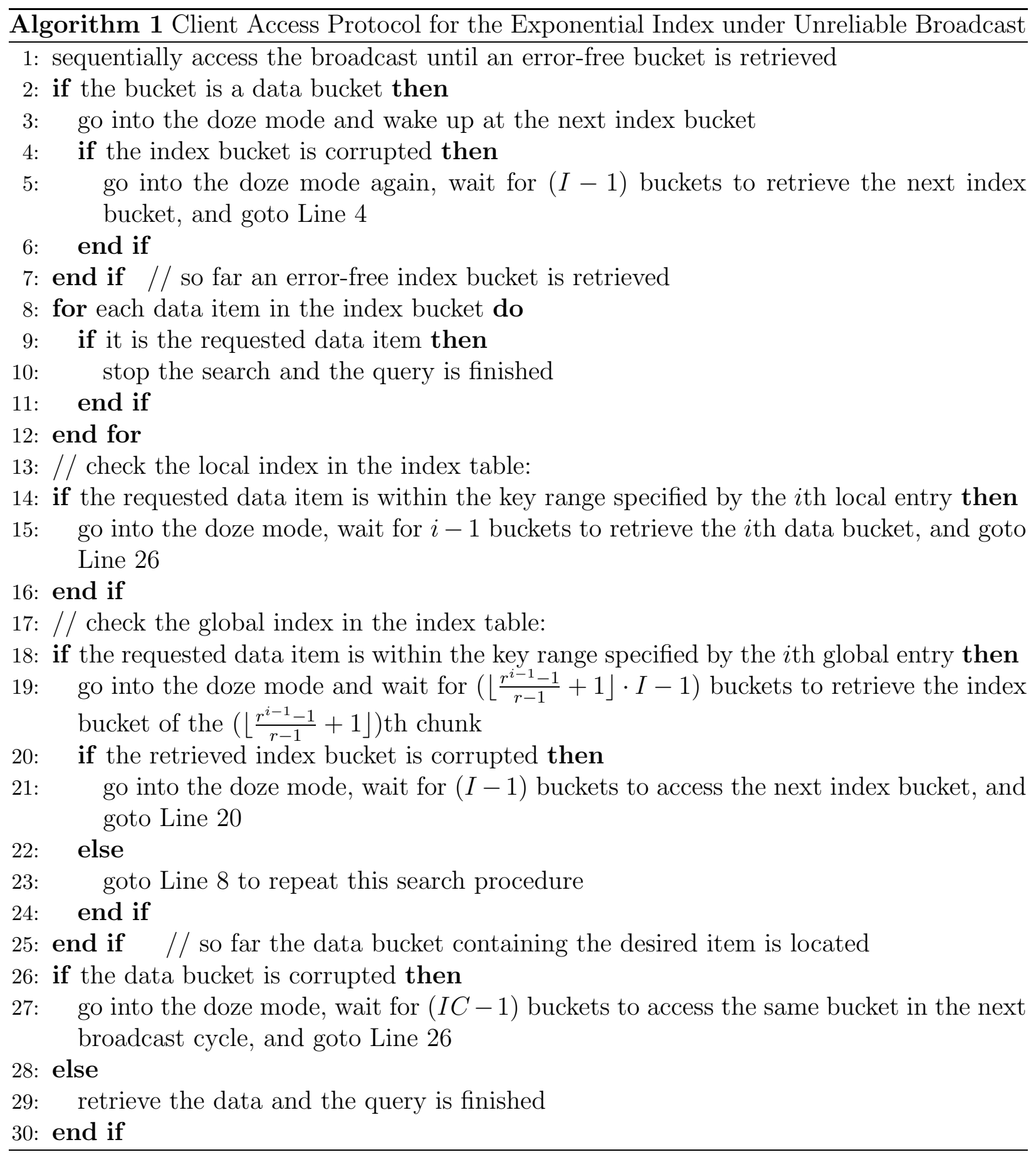

chunk size $I$. These two parameters offer the exponential index great flexibility in tuning access latency against tuning time. In general, the number of index entries and hence the indexing overhead increases with decreasing index base $r$, and the tuning time decreases with $r$. Moreover, the larger the chunk size $I$, the less the tuning time but the longer the initial index probing time. A detailed performance analysis is provided in the next section. 


\begin{tabular}{ll}
\hline Notation & Description \\
\hline$N$ & number of data items \\
$B$ & capacity of a data bucket without an index \\
$B^{\prime}$ & capacity of a data bucket with an index \\
$p$ & probability of a bucket being corrupted \\
$s_{o}$ & size of a data item \\
$s_{e}$ & size of an index entry \\
$I$ & chunk size, i.e., \# buckets in a data chunk \\
$r$ & index base \\
$C$ & number of chunks in a bcast \\
$s_{i}$ & size of index table for each chunk \\
$n_{i}$ & number of entries in an index table \\
$n_{b}$ & number of local index entries for local buckets \\
$n_{c}$ & number of global index entries for data chunks \\
$E(d)$ & average access latency \\
$E(t)$ & average tuning time \\
$O(t)$ & worst tuning time \\
\hline
\end{tabular}

Table 1: Summary of Notations

\subsection{Performance Analysis}

This section analyzes the access latency and tuning time of the exponential index. We assume that the access probabilities of data items are uniformly distributed and the initial points to tune in the broadcast channel are randomly distributed over the bcast. Table 1 summarizes the notations used in the analysis.

Let $B$ denote the number of data items that a data bucket can hold. Since an index table needs to occupy the space used to store data items, fewer items can be accommodated by a bucket with an index table. Let $B^{\prime}$ denote the number of items such a bucket can hold. The value of $B^{\prime}$ is a function of the parameters of $I$ and $r$. Note that $B^{\prime}$ is an integer and $r$ is a real number. An arbitrary $r$ may not result in an index table of a size equal to a multiple of the data item size. Since the tuning time generally decreases with the index base $r$, it is desirable to adjust $r$ according to $B^{\prime}$ to fully exploit the available space for an index table.

Given $B$ and $B^{\prime}$, the number of entries in an index table follows:

$$
n_{i} \leq \frac{\left(B-B^{\prime}\right) \cdot s_{o}}{s_{e}},
$$

where $s_{o}$ and $s_{e}$ are the sizes of a data item and an index entry, respectively. 
Since a data chunk consists of $I$ buckets, the number of local index entries is simply given by: $n_{b}=I-1$. Thus, we obtain the number of global index entries:

$$
n_{c}=n_{i}-n_{b} \leq \frac{\left(B-B^{\prime}\right) \cdot s_{o}}{s_{e}}-I+1
$$

As a data chunk consists of $I-1$ buckets without index tables and one bucket with an index table, it can hold a total of $B(I-1)+B^{\prime}$ data items. Hence, the number of data chunks in a bcast is given by:

$$
C=\left\lceil\frac{N}{B(I-1)+B^{\prime}}\right\rceil
$$

The index table in each chunk indexes all the other chunks in a bcast; thus, we must have

$$
\sum_{i=1}^{n_{c}} r^{i-1}=\frac{r^{n_{c}}-1}{r-1} \geq C-1
$$

Therefore, given $B^{\prime}$ and $I$, the smallest value of $r$ can be obtained by numerically solving the following inequality:

$$
r^{n_{c}}+(1-C) r+C-2 \geq 0
$$

Now we derive the average access latency and tuning time given $B^{\prime}$ and $I$. The average access latency is obtained as follows (see the Appendix for detailed derivation):

$$
E(d)=\frac{I C}{2}+1+\frac{I C \cdot p}{1-p}+\left(\frac{I}{2}+\frac{I C \cdot p}{1-p}\right) \cdot \frac{B(I-1)}{B(I-1)+B^{\prime}} .
$$

The average tuning time is given by (see the Appendix for detailed derivation):

$$
E(t)=\frac{2 I-1}{I(1-p)}+\frac{1}{C}\left(t(0)+\sum_{l=1}^{C-1}\left(t(l)+\frac{t(C-1) \cdot p}{1-p}\right)\right)+\frac{B(I-1)}{\left(B(I-1)+B^{\prime}\right) \cdot(1-p)}
$$

where

$$
t(l)= \begin{cases}0, & \text { if } l=0 \\ t(l-x) \cdot(1-p)+t(l-1) \cdot p+1, & \text { if } l>0\end{cases}
$$

where $x$ is the maximum value less than or equal to $l$ in the set of $\left\{1,2,\lfloor r+2\rfloor, \cdots,\left\lfloor\frac{r^{n_{c}-1}-1}{r-1}\right\rfloor+1\right\}$. 
From (5) and (7), it is not difficult see that with the same value of $B^{\prime}$, the smaller is the value of $r$, the less is the average tuning time in general. Therefore, in performance optimization and tuning, we examine only the smallest values of $r$ that result in an index table whose size is a multiple of the data item size, rather than testing all possible values of $r$.

To have more intuition on tuning time, we also derive the worst tuning time, assuming the broadcast is error-free. ${ }^{4}$ Suppose the client initially tunes into data chunk $A$ and is interested in some data item in chunk $T$ (see Figure 3). The initial search space is $C$ (approximately $\left.\frac{r^{n_{c}}-1}{r-1}\right)$ buckets. According to the index table in $A$, the search will be guided to a certain range of sequential data chunks, whose size is at most $r^{n_{c}-1}$ chunks (when $T$ falls in the last index entry). Thus, the search space is reduced by a factor of at least

$$
\frac{\frac{r^{n_{c}}-1}{r-1}}{r^{n_{c}-1}}=\frac{r-r^{1-n_{c}}}{r-1} \approx \frac{r}{r-1} .
$$

Then, the client will access the first chunk in the refined search space (e.g., chunk $B$ in Figure 3) and trim the search space again by a factor of at least $\frac{r}{r-1}$ through examination of $B$ 's index table. The procedure is repeated until the refined search space contains one data chunk only. Therefore, at most $\left\lceil\log _{\frac{r}{r-1}}(C-1)\right\rceil+1$ buckets are accessed to reach the target chunk. If a chunk contains more than one bucket, we might need one more bucket access to probe the first index bucket and another one to locate the desired data item after reaching the target chunk. Therefore, the tuning time is bounded by:

$$
O(t)= \begin{cases}\left\lceil\log _{\frac{r}{r-1}}(C-1)\right\rceil+1, & \text { if } I=1 \\ \left\lceil\log _{\frac{r}{r-1}}(C-1)\right\rceil+3, & \text { if } I>1\end{cases}
$$

\subsection{Performance Tuning}

As mentioned before, tuning time and access latency are two conflicting performance measures; they cannot be minimized at the same time. To cater for different application scenarios, we need tunable indexing structures that optimize either the tuning time or the access latency with a certain performance requirement on the other metric. The proposed expo-

\footnotetext{
${ }^{4}$ The worst tuning time is $\infty$ when considering link errors.
} 
nential index can be employed to serve this purpose. Specifically, we are interested in tuning the performance along two dimensions:

- Latency-bounded tuning: Given a limit $L$ on the average access latency, how can the parameters (i.e., $r$ and $I$ ) of the exponential index be tuned to obtain the minimum tuning time?

- Tuning-time bounded tuning: Given a limit $T$ on the average tuning time, how can the parameters of the exponential index be tuned to achieve the shortest access latency?

Based on the analysis presented in the last section, the optimal solutions to the above two problems can be obtained by searching the optimal values of $B^{\prime}$ (recall that $r$ is a function of $B^{\prime}$ as shown by (2), (3), and (5)) and $I$. Thus, the latency-bounded tuning problem is defined as follows:

$$
\begin{array}{r}
\min _{I=\left\{1,2, \cdots,\left\lceil\frac{N}{B}\right\rceil\right\}, B^{\prime}=\left\{0,1, \cdots,\left\lfloor B-\frac{I \cdot s_{e}}{s_{o}}\right\rfloor\right\}} E(t), \\
\text { s. t. } E(d) \leq L .
\end{array}
$$

The tuning-time-bounded tuning problem is defined as follows:

$$
\begin{array}{r}
\min _{I=\left\{1,2, \cdots,\left\lceil\frac{N}{B}\right\rceil\right\}, B^{\prime}=\left\{0,1, \cdots,\left\lfloor B-\frac{I \cdot s_{e}}{s_{o}}\right\rfloor\right\}} E(d), \\
\text { s. t. } E(t) \leq T .
\end{array}
$$

It is easy to see that these two search problems have a worst-case time complexity of $\mathcal{O}\left(\frac{N}{B} \cdot B\right)=\mathcal{O}(N)$.

\section{Performance Evaluation}

This section evaluates the performance of the proposed exponential index. We developed a simulator based on ns-2 to simulate the GPRS wireless network $[2,11]$. We would like to compare the exponential index against the state-of-the-art indexes (i.e., the distributed tree [10] and the flexible index [9] enhanced for unreliable broadcast [23]) and to investigate its ability to adjust the trade-off between access latency and tuning time. 
In the simulation, a broadcast server and a client are simulated, and the user requests are sequentially issued and processed by the client. ${ }^{5}$ We set the system parameters similar to those in $[9,10,23]$. The database size is set at 30,000 items. The link error probability ranges from 0 to $10 \%$. Flat broadcast is employed to broadcast the data items. Without loss of generality, we assume that the access distribution over the data items is uniform. ${ }^{6}$ For the exponential index and the flexible index, an index entry contains a key value only; hence, its size is set to 4 bytes. For the distributed tree, the index entry size is set to 8 bytes since it contains a key value as well as the offset to the bucket containing the key value. We have evaluated different combinations of item size $s_{o}$ and bucket capacity $B$. Due to space limitations, we report the results for two informative settings only, i.e., 1) $s_{o}=16$ bytes, $B=80$ (denoted as "S-16 B-80"); and 2) $s_{o}=128$ bytes, $B=10$ (denoted as "S-128 B-10"). Recall that each data bucket includes an offset to the beginning of the next index bucket. For simplicity, we omit this overhead since it is very small and exists in all the indexing schemes under investigation. The system parameter settings are summarized in Table 2 .

We compare the indexing schemes in terms of the tuning time and access latency, both of which are measured in the unit of buckets. To make a clear comparison, the access latency of an indexing scheme shown in the results is normalized by the latency of a non-index scheme, i.e., $\left\lceil\frac{N}{2 B}\right\rceil$. The results reported for all indexes under investigation were obtained from simulation. We have also calculated the results for the exponential index based on the analysis presented in Section 3; the simulation results match the analytical results, which confirms the correctness of the analysis.

\begin{tabular}{||l|l|l||l|}
\hline Parameter & Setting & Parameter & Setting \\
\hline$N$ & 30,000 & $B$ & 10,80 \\
\hline$s_{o}$ & 16,128 bytes & $s_{e}$ & 4 bytes \\
\hline$p$ & $0-10 \%$ & & \\
\hline
\end{tabular}

Table 2: Parameter Settings

\footnotetext{
${ }^{5}$ Note that the data access rate will not have an impact on the overall performance.

${ }^{6}$ With flat broadcast, the average performance of the indexes would remain the same no matter what the data access pattern is.
} 


\subsection{Comparison with the Distributed Tree Index}

This set of experiments compares the proposed exponential index to the distributed tree, which is a non-flexible scheme. To compare the tuning time, we first measure the performance of the distributed tree given the default system setting; we then obtain the best tuning time for the exponential index by tuning the index base and chunk size such that its access latency is no higher than that of the distributed tree. Figures 5a shows the average tuning time as a function of link error probability. As expected, the tuning time increases with increasing error probability for both index schemes. The exponential index outperforms the distributed tree by $25 \%-42 \%$.

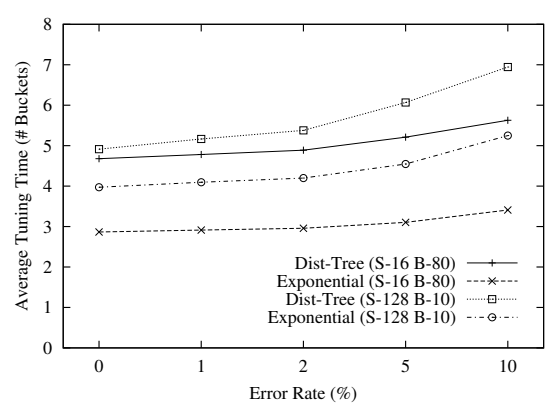

(a) Average Tuning Time

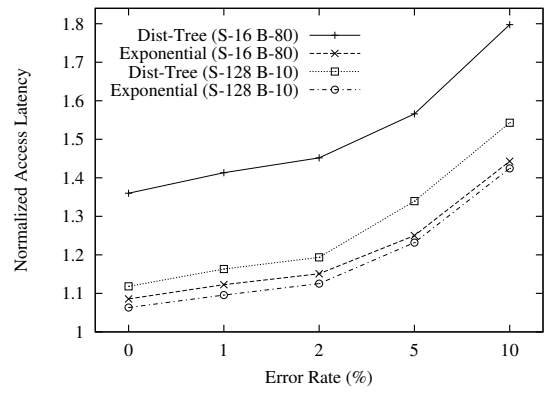

(b) Normalized Access Latency

Figure 5: Exponential vs. Distributed Tree Index

Similarly, to compare the access latency, we tune the parameters of index base and chunk size to obtain the best result for the exponential index while making sure its tuning time is no worse than that of the distributed tree. As shown in Figure 5b, the exponential index achieves a better performance than the distributed tree for all cases.

\subsection{Comparison with the Flexible Index}

This section compares the proposed exponential index to the flexible index in terms of their effectiveness in reducing the tuning time. Note that these two schemes have a similar performance for a local data search within a chunk. Therefore, to facilitate the comparison, we set the chunk size to one bucket to observe their performance differences for global index search across data chunks. For the exponential index, we adjust the index base $r$ such that 


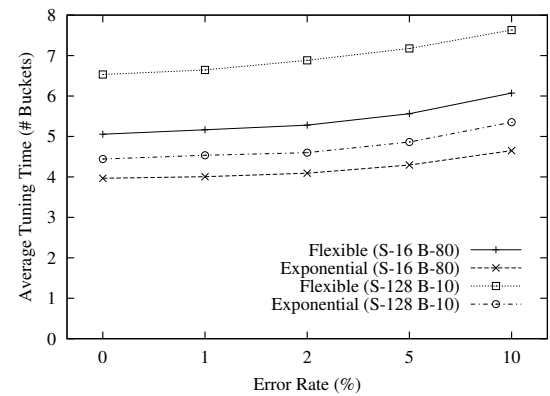

(a) Average Tuning Time

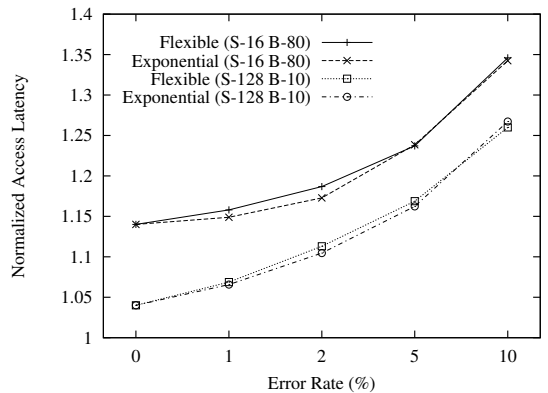

(b) Normalized Access Latency

Figure 6: Exponential vs. Flexible Index (Chunk Size=1 Bucket)

it achieves a similar access latency to that of the flexible index. Figures $6 \mathrm{a}$ and $6 \mathrm{~b}$ show the average tuning time and the normalized access latency, respectively.

As shown in Figure 6, with the same (or even less) access latency (Figure 6b), the exponential index consistently outperforms the flexible index in terms of the tuning time (Figure 6a). The improvement is more significant for the setting of item size 128 bytes, bucket capacity 10 (i.e., S-128, B-10) than the setting of item size 16 bytes, bucket capacity 80 (i.e., S-16, B-80). This can be explained as follows. The flexible index employs a binary control index, which blindly incurs overhead without considering the available space. Thus, the larger the item size, the higher the probability of leaving large internal fragments. On the other hand, the exponential index adjusts the parameter of $r$ according to the available space for indexing. Hence, with a large item size, it can fully utilize the large item space to achieve a better performance.

\subsection{Flexibility of the Indexes}

This section investigates the indexes' ability to adjust the trade-off between access latency and tuning time. First, we look at the tuning-time-bounded tuning problem. It is desirable that the longer is the tuning time allowed, the shorter is the access latency achieved. Figures $7 \mathrm{a}$ and $7 \mathrm{~b}$ respectively show the results for the settings of S-16, B-80 and S-128, B-10 under the default link error probability 1\%. As expected, the distributed tree is not flexible: it is impossible for it to achieve a tuning time shorter than 4.8 buckets, and the latency 


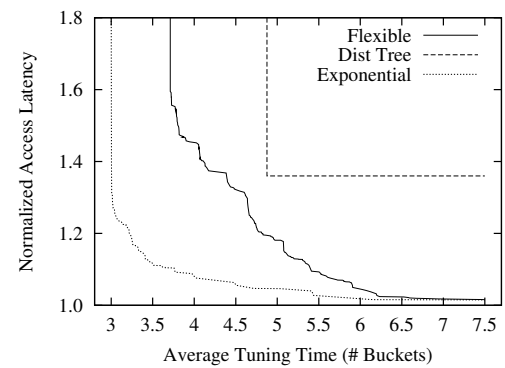

(a) Link Error $=1 \%, \mathrm{~S}-16, \mathrm{~B}-80$

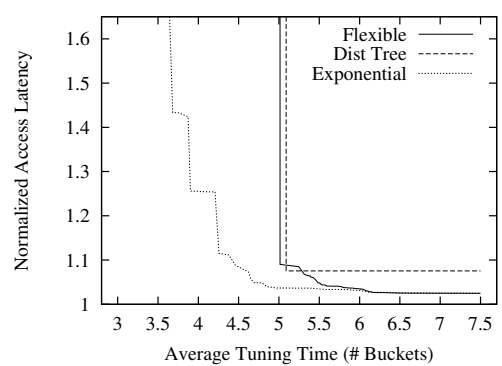

(b) Link Error $=1 \%$, S-128, B-10

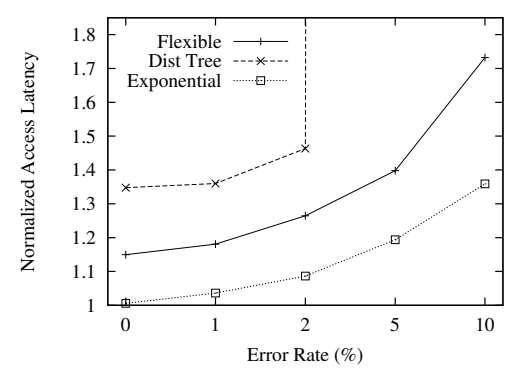

(c) Link Error $=0-10 \%$, S-16, B-80

Figure 7: Access Latency with Bounded Tuning Time

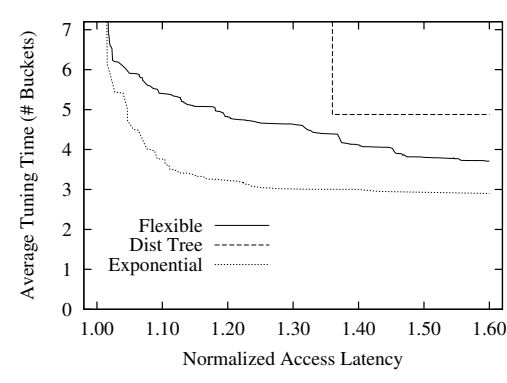

(a) Link Error $=1 \%$, S-16, B-80

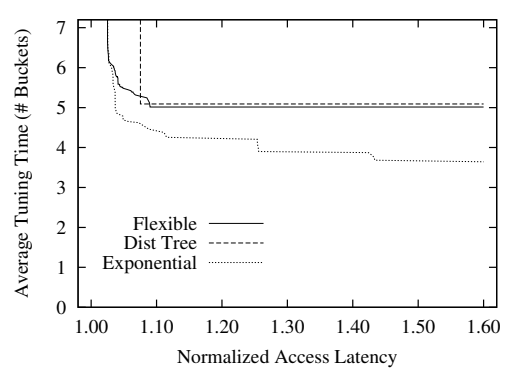

(b) Link Error $=1 \%$, S-128, B-10

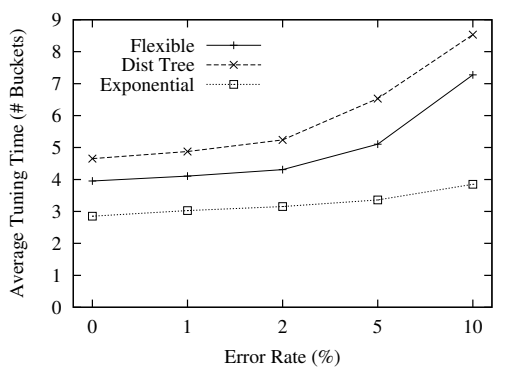

(c) Link Error $=0-10 \%$, S-16, B-80

Figure 8: Tuning Time with Bounded Access Latency

remains the same after this point. While the flexible index is able to trade access latency for tuning time, obviously the exponential index performs even better. With the same tuning time requirement, the exponential index achieves a shorter (or the same) access latency. For a similar reason to that explained in Section 4.2, the improvement of the exponential index over the flexible index is more remarkable for the setting with a larger item size.

To examine the indexes' resilience to link errors, we show in Figure 7c the achieved latency with a bounded tuning time of 5.0 under a variety of link error probabilities. As can be seen, the performance improvement of the exponential index over the other two indexes becomes more significant (e.g., from $12 \%$ to $20 \%$ against the flexible index) with increasing error probability, implying the exponential index is more resilient to link errors. This confirms our claim that the exponential index recovers an index search from a link error more quickly (as discussed in Section 3).

Next, we examine the latency-bounded tuning problem. We expect to achieve a shorter 
tuning time by tolerating a longer latency. As shown in Figures 8a and 8b, the distributed tree obtains a better performance than the flexible index only at normalized access latencies of 1.07-1.09 for the setting of S-128, B-10. The exponential index performs the best throughout the range of bounded latencies tested.

Figure 8c shows the tuning time under a variety of link error probabilities when the access latency is bounded at 1.4. Once again, the exponential index demonstrates a stronger resilience to link errors. As the link error probability is increased from 0 to $10 \%$, the performance improvement of the exponential index over the distributed index and the flexible index increases from $36 \%$ to $53 \%$ and $26 \%$ to $44 \%$, respectively.

\section{Extensions}

The previous sections have focused on clustered broadcast, which is capable of indexing the primary attribute in flat broadcast. This section extends the proposed exponential index to non-clustered broadcast, which is useful for indexing secondary attributes and skewed broadcast. In addition, we leverage the idea of index caching to further improve the access performance.

\subsection{Non-Clustered Broadcast}

As discussed in Section 2, for non-clustered broadcast, a bcast can be partitioned into a number of clustered segments (i.e., meta-segments). The number of meta-segments in a bcast for an attribute is called the scattering factor (denoted by $M$ ) [10]. Without loss of generality, we assume the items are sorted in ascending order of the attribute values in each meta-segment. Similar to clustered broadcast, the exponential index can be applied to each meta-segment. Instead of indexing a whole bcast, each index table for non-clustered broadcast covers the buckets up to the farthest one in the next meta-segment whose attribute value is less than that of the current bucket. In the example shown in Figure 9, the index table in bucket 2 indexes buckets 3-5, and the index table in bucket 10 indexes buckets $11-13$.

The client access protocol remains the same except that a query continues to search the 


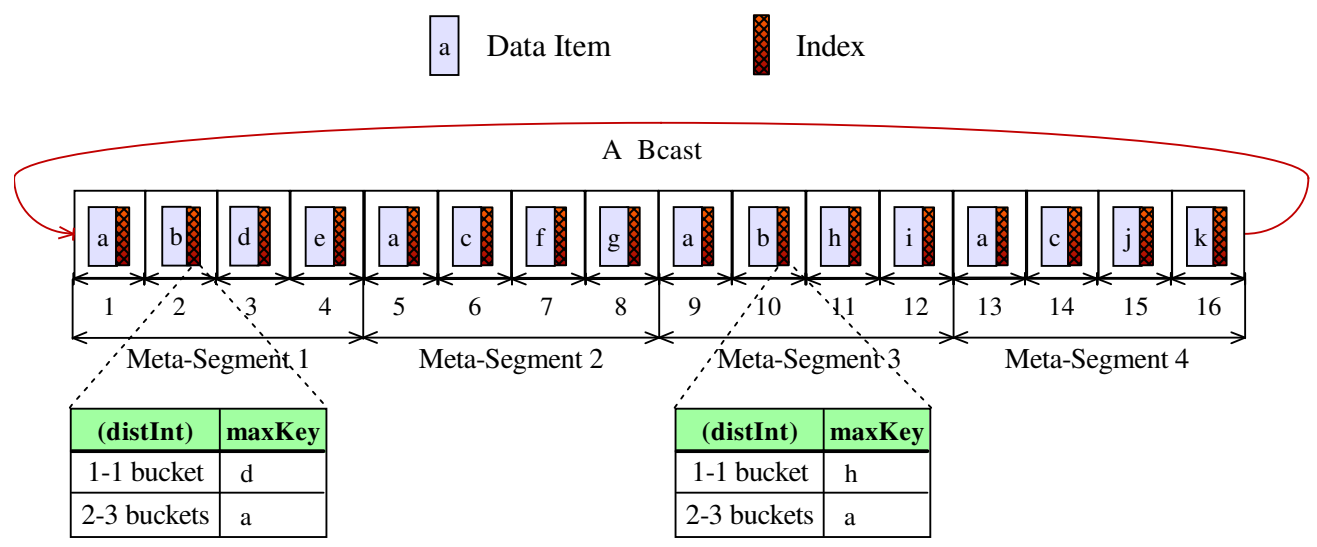

Figure 9: Indexing Non-Clustered Broadcast $(r=2, I=1)$

next segment if the target item is not found in the current meta-segment. The maximum number of meta-segments to be searched is $M$. Thus, based on (10), the tuning time of a query is bounded by $\mathcal{O}\left(M \log _{\frac{r}{r-1}} S\right)$, where $S$ is the number of chunks in a meta-segment.

We now evaluate the flexibility of the exponential index for non-clustered broadcast. We simulate a broadcast-disk system [1] which consists of three disks with $800,1,600$, and 23,600 data items, respectively. The three disks are interleaved in a bcast but rotate at different speeds: the first disk rotates at a speed twice as fast as the second one and four times as fast as the third disk. In other words, the items in the first, second, and third disk are broadcast 4, 2, and 1 time(s) respectively in each bcast. The resulting bcast has four meta-segments, each of which contains 7,500 clustered items. The access probability of each item is set proportional to its broadcast frequency. The distributed tree and the flexible index are included for comparison. For both of the index schemes, an index is built for each meta-segment; similar to the exponential index, if the item of interest is not found in the current meta-segment, the search continues in the next meta-segment until the item is retrieved.

As shown in Figure 10, the exponential index has the best performance among all indexes under investigation. Compared with the clustered broadcast case (Figures 7a and 8a), the improvement here is more significant. For example, in tuning-time bounded tuning, the exponential index achieves a tuning time of 4.9 buckets at a normalized latency of 1.6, whereas the flexible index cannot get a tuning time shorter than 7.6 buckets (cf. 3.0 vs. 3.7 


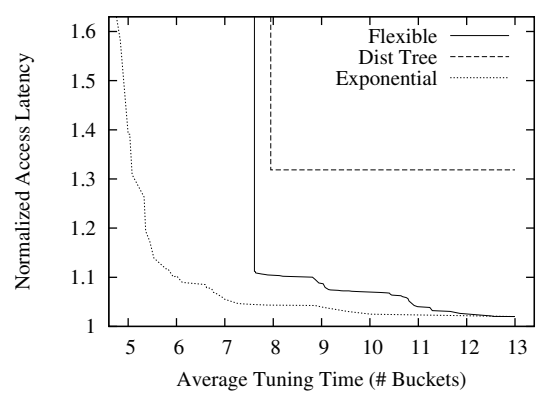

(a) Tuning-Time Bounded Tuning

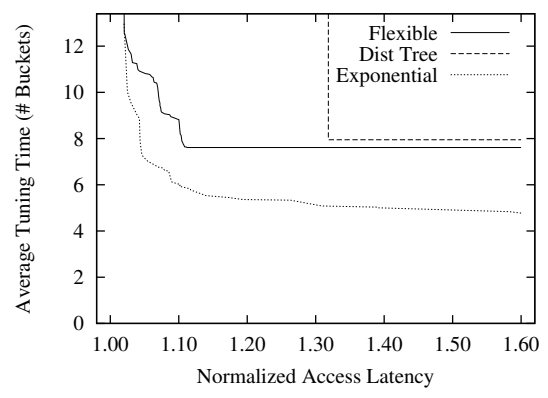

(b) Access-Latency Bounded Tuning

Figure 10: Flexibility of the Indexes for Non-Clustered Broadcast (S-16, B-80, Link Error $=1 \%$ )

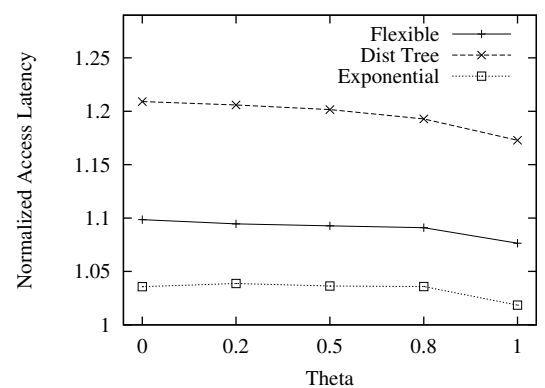

(a) Tuning-Time Bounded Tuning

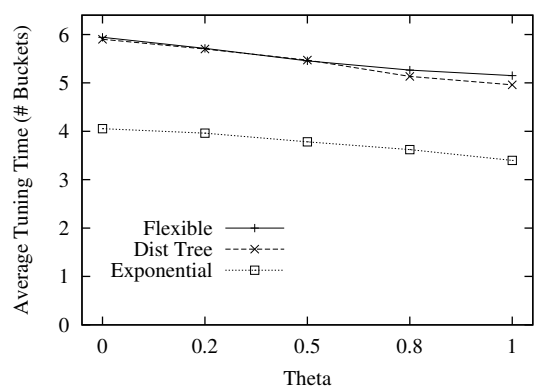

(b) Access-Latency Bounded Tuning

Figure 11: Impact of Skewed Access for Non-Clustered Broadcast (S-16, B-80, Link Error $=1 \%$ )

buckets in Figure 7a). This is mainly because the exponential index allows the current metasegment to index into the next meta-segment to support continuous search, thus improving search efficiency; in contrast, the distributed tree and the flexible index constrain the indexing space within a meta-segment only and, hence, when the item is not found in the current meta-segment, the search has to restart from the index root in the next meta-segment.

To evaluate the impact of skewed data access, a Zipf distribution (with a skewness parameter $\theta$ ) is used to simulate client access behavior [26]. The broadcast-disk system described earlier is employed to generate broadcast program. Figure 11 shows the results under different settings of $\theta$. We set the bounded tuning time at 11 for Figure 11a and the bounded access latency at 1.8 for Figure 11b. It can be observed the exponential index consistently outperforms the distributed tree and the flexible index. This demonstrates that the exponential index is robust to skewed data access. 


\subsection{Index Caching}

We now discuss how to cache the index to further improve data access performance. In particular, we shall show that the exponential index can benefit more performance advantages from index caching than the other indexes.

The client access protocol with index caching is similar to that without it. Upon a data request, the client first tunes into the broadcast and retrieves the first index bucket by following Algorithm 1. If the index (table) is not cached yet, it is cached on the client. Then, it follows the exponential index to determine which index bucket to access next. If the next index (table) is in the cache, it can be accessed immediately from the cache. Otherwise, as usual, the client tunes into the broadcast to retrieve it. An issue here is how to tell if the next index (table) is cached or not, or equivalently how to figure out the id of the next index (table). Recall that each bucket contains a sequentially increased id number. As such, the id of the next index (table) can be computed from the id of the current and the indexing distance. The saving for index data retrieval due to index caching can also be achieved by the distributed tree and the flexible index.

Yet the exponential index can do more than that thanks to its nice data structure. Since multiple search trees are embedded in the exponential index, we can make use of this feature to further refine the search space with caching. We take one example to illustrate this additional advantage. Assume again the broadcast program is as shown in Figure 2. To retrieve item "SUNW" starting from the "DELL," without index caching, the client accesses buckets 1, 5, 7, and 8, as discussed in Section 3.1. Now suppose that the index table of bucket 3 is cached. By first accessing bucket 1, we know that the desired item must be stored in buckets 5 through 8 . Moreover, we figure out from the cached index table that the item must be in buckets 7 through 10 . Hence, the desired item should lie in buckets 7 and 8 . Therefore, we next access buckets 7 and 8 to retrieve the item "SUNW." Compared to the no-caching case, one bucket access can be saved. With more indexes cached, more performance improvement can be expected. The index cache access algorithm is formally described in Algorithm 2.

To focus on the performance of index caching, we assume there are no index updates 

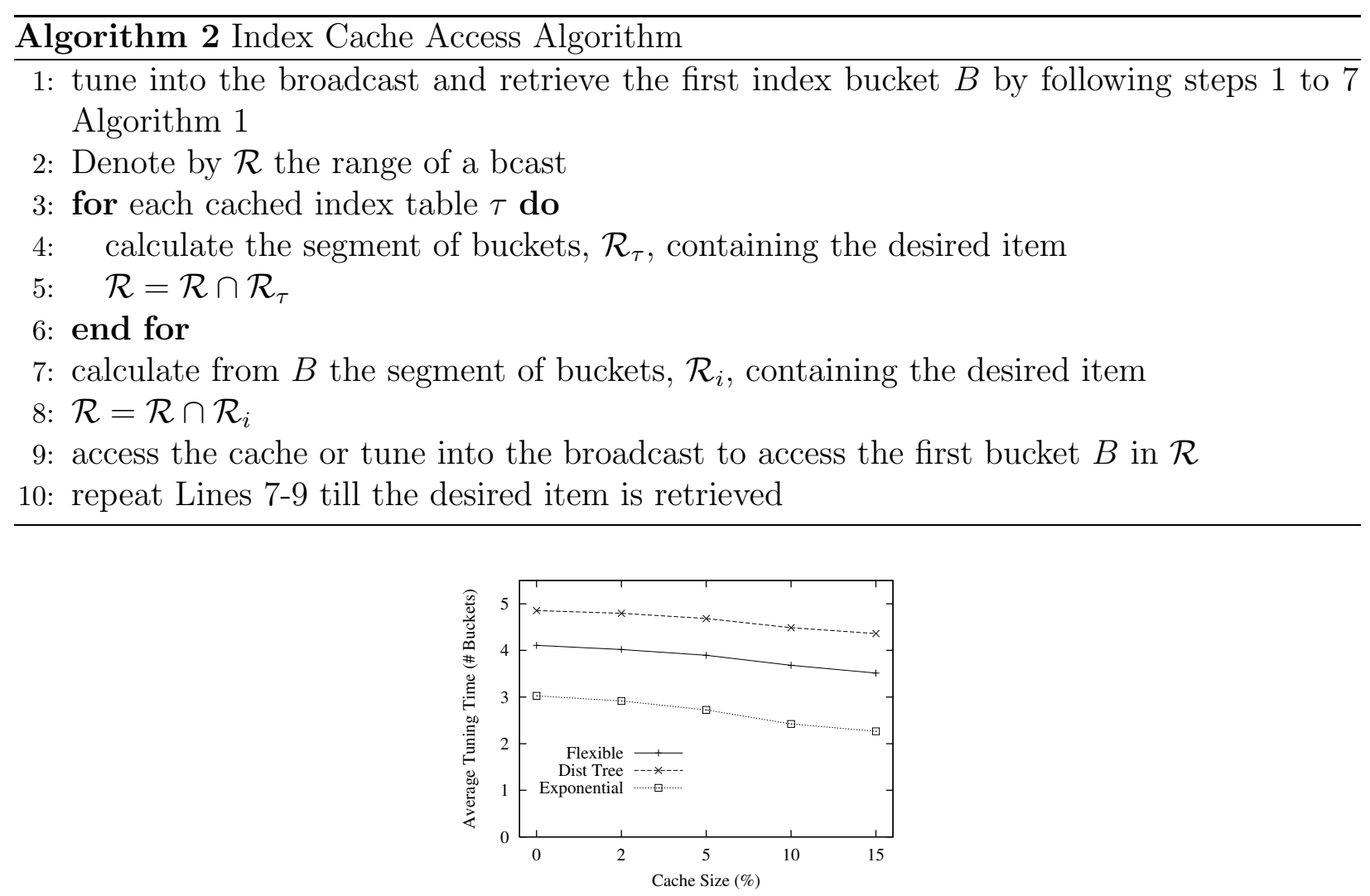

Figure 12: Performance as a Function of Cache Size (S-16, B-80, Link Error=1\%)

in the experiments. ${ }^{7}$ We employ LRU as the cache replacement policy. Figure 12 shows the tuning time as a function of cache size, where the flat broadcast is employed and the bounded access latency is set at 1.4. The cache size is represented as a percentage of the size of the distributed tree index. As observed, as the cache size is increased from 0 to $15 \%$, all schemes improve their performance. Among the three schemes, the exponential index reduces the tuning time most significantly as expected (i.e., $24 \%$ vs. $11 \%$ for distributed tree and $15 \%$ for flexible index). As a result, its performance gain over the distributed tree and the flexible index is increased from $62 \%$ to $96 \%$ and from $37 \%$ to $56 \%$, respectively.

\footnotetext{
${ }^{7}$ The cache consistency issue for data broadcast has been well studied in the literature. This is out of the scope of this paper however; interested readers are referred to [3, 22].
} 


\section{Conclusions}

This paper has investigated the use of air indexing techniques to improve the efficiency of energy consumption on mobile devices in an unreliable broadcast system. We have proposed a novel parameterized index scheme called the exponential index. It has a linear yet distributed structure which suits the broadcast environment very well. The distributed property of the exponential index enables a search to start immediately from an arbitrary index table in the broadcast as well as to restart quickly in case of a link error. The energy consumption of mobile clients is also very efficient (i.e., the tuning time is logarithmically proportional to the bcast length). Moreover, the access latency and tuning time of the exponential index can be adjusted by two tuning knobs: index base and chunk size.

We have provided an analytical model to derive the access latency and tuning time of the exponential index and analyzed how to minimize the access latency (or tuning time) with a bounded tuning time (or access latency). We have demonstrated via simulations that 1) the exponential index substantially outperforms two state-of-the-art air indexing schemes;

2 ) it is more resilient to link errors; 3) it benefits more performance advantages from index caching; and 4) it achieves a greater flexibility in adjusting the trade-off between access latency and tuning time.

There are a number of issues that deserve further study. The proposed index exponentially partitions the indexing space, yet the optimal partitioning remains an open problem. The exponential index does not differentiate the performance requirements of individual clients or items. We plan to investigate client based and item based flexible indexes. In addition, we are interested in exploring the research issues of balancing access latency and tuning time in a multi-channel data broadcast environment.

\section{Acknowledgments}

The authors would like to thank the anonymous reviewers for their constructive comments. Prof. Michael Franklin at UC Berkeley offered many valuable suggestions on an earlier version of this paper. Jianliang Xu's work was partially supported by grants from the 
Research Grants Council of the Hong Kong SAR, China (Project Nos. HKBU 2115/05E and HKBU FRG/04-05/II-26). Xueyan Tang's work was supported in part by a grant from Nanyang Technological University. Wang-Chien Lee's work was supported in part by National Science Foundation grant IIS-0328881.

\section{References}

[1] S. Acharya, R. Alonso, M. Franklin, and S. Zdonik. Broadcast disks: Data management for asymmetric communications environments. In Proceedings of ACM SIGMOD Conference on Management of Data, pages 199-210, San Jose, CA, May 1995.

[2] C. Bettstetter, H.-J. Vogel, and J. Eberspacher. GSM phase 2+ general packet radio service GPRS: Architecture, protocols, and air interface. IEEE Communications Surveys, $2(3), 1999$.

[3] G. Cao. A scalable low-latency cache invalidation strategy for mobile environments. IEEE Transactions on Knowledge and Data Engineering (TKDE), 15(5):1251-1265, September/October 2003.

[4] M.-S. Chen, K.-L. Wu, and P. S. Yu. Optimizing index allocation for sequential data broadcasting in wireless mobile computing. IEEE Transactions on Knowledge and Data Engineering (TKDE), 15(1):161-173, January/February 2003.

[5] C.-L. Hu and M.-S. Chen. Dynamic data broadcasting with traffic awareness. In Proceedings of IEEE International Conference on Distributed Computing Systems (ICDCS'O2), pages 112-119, Vienna, Austria, July 2002.

[6] Q. L. Hu, W.-C. Lee, and D. L. Lee. Power conservative multi-attribute queries on data broadcast. In Proceedings of the 16th International Conference on Data Engineering (ICDE'00), pages 157-166, San Diego, CA, February 2000.

[7] Q. L. Hu, W.-C. Lee, and D. L. Lee. A hybrid index technique for power efficient data broadcast. Distributed and Parallel Databases (DPDB), 9(2):151-177, March 2001. 
[8] W. C. Huffman and V. Pless. Fundamentals of Error-Correcting Codes, Cambridge University Press, 2003.

[9] T. Imielinski, S. Viswanathan, and B. R. Badrinath. Power efficient filtering of data on air. In Proceedings of the 4 th International Conference on Extending Database Technology (EDBT'94), pages 245-258, Cambridge, UK, March 1994.

[10] T. Imielinski, S. Viswanathan, and B. R. Badrinath. Data on air - Organization and access. IEEE Transactions on Knowledge and Data Engineering (TKDE), 9(3):353-372, May/June 1997.

[11] R. Jain. GPRS simulations using ns - network simulator. Master Thesis, Dept. of Electrical Enngineering, India Institue of Technology - Bombay, June 2001. Source code available at http://www.isi.edu/nsnam/ns/ns-contributed.html.

[12] K. C. K. Lee, H. V. Leong, and A. Si. Semantic data broadcast for a mobile environment based on dynamic and adaptive chunking. IEEE Transactions on Computers, 51(10):1253-1268, October 2002.

[13] V. C. S. Lee, J. K. Ng, J. Y. P. Chong, and K.-W. Lam. Maintaining temporal consistency in broadcast environments. In Proceedings of 5th IEEE International Conference on Mobile Data Management (MDM'04), Berkeley, CA, January 2004.

[14] V. Liberatore. Caching and scheduling for broadcast disk systems. Technical Report 98-71, Institute for Advanced Computer Studies, University of Maryland at College Park (UMIACS), December 1998.

[15] V. Liberatore. Multicast scheduling for list requests. In Proceedings of IEEE INFOCOM'02, pages 1129-1137, New York, NY, June 2002.

[16] DirectBand Network. Microsoft Smart Personal Objects Technology (SPOT). [Online]. Available: http://www.microsoft.com/resources/spot/. 
[17] E. Pitoura and P. Chrysanthis. Exploiting versions for handling updates in broadcast disks. In Proceedings of Very Large Data Bases (VLDB'99), pages 114-125, Edinburgh, Scotland, 1999.

[18] Q. Ren, M. H. Dunham, and V. Kumar. Semantic caching and query processing. IEEE Transactions on Knowledge and Data Engineering (TKDE), 15(1):192-210, January/February 2003.

[19] N. Shivakumar and S. Venkatasubramanian. Energy-efficient indexing for information dissemination in wireless systems. ACM/Baltzer Journal of Mobile Networks and Applications (MONET), 1(4):433-446, December 1996.

[20] StarBand. [Online]. Available at http://www.starband.com/.

[21] Hughes Network Systems. DIRECWAY homepage. [Online]. Available at http://www.direcway.com/.

[22] K. L. Tan, J. Cai, and B. C. Ooi. An evaluation of cache invalidation strategies in wireless environments. IEEE Transactions on Parallel and Distributed Systems (TPDS), 12(8): 789-807, August 2001.

[23] K. L. Tan and B. C. Ooi. On selective tuning in unreliable wireless channels. Journal of Data and Knowledge Engineering (DKE), 28(2):209-231, November 1998.

[24] K. L. Tan and J. X. Yu. Energy efficient filtering of nonuniform broadcast. In Proceedings of the 16th International Conference on Distributed Computing Systems (ICDCS'96), pages 520-527, Hong Kong, May 1996.

[25] M. A. Viredaz, L. S. Brakmo, and W. R. Hamburgen. Energy management on handheld devices. ACM Queue, 1(7):44-52, October 2003.

[26] J. Xu, Q. L. Hu, W.-C. Lee, and D. L. Lee. Performance evaluation of an optimal cache replacement policy for wireless data dissemination. IEEE Transactions on Knowledge and Data Engineering (TKDE), 16(1):125-139, January 2004. 
[27] J. Xu, W.-C. Lee, and X. Tang. Exponential Index: A parameterized distributed indexing scheme for data on air. In Proceedings of the 2nd ACM/USENIX International Conference on Mobile Systems, Applications, and Services (MobiSys'04), pages 153-164, Boston, MA, June 2004.

[28] J. Xu, B. Zheng, W.-C. Lee, and D. L. Lee. Energy efficient index for querying locationdependent data in mobile broadcast environments. In Proceedings of the 19th IEEE International Conference on Data Engineering (ICDE'03), pages 239-250, Bangalore, India, March 2003. 


\section{Supplemental Material}

\section{Appendix: Derivation of Average Access Latency and Tuning Time}

First, we derive the average access latency given $B^{\prime}$ and $I$. Recall that the data access is divided into three phases (Algorithm 1): initial probe (lines 1-7), index search (lines 8-25), and data retrieval (lines 26-30). With an error-free broadcast, the average latency until the moment when the index bucket of the chunk containing the desired data item (termed as the target index bucket) is received is $\frac{I}{2}+\frac{I(C-1)}{2}+1=\frac{I C}{2}+1$ buckets. However, if the target index bucket is corrupted, we will have to delay a bcast (i.e., IC buckets) to get its next broadcast instance. In general, assuming a link error probability of $p$, the probability of the target index bucket being corrupted continuously for $i$ times is $p^{i}(1-p)$; in this case, an additional delay of $i \cdot I C$ is incurred. Thus, the average access latency for the phases of initial probe and index search is given by:

$$
E\left(d_{p i}\right)=\frac{I C}{2}+1+\sum_{i=0}^{\infty} p^{i}(1-p) \cdot i \cdot I C=\frac{I C}{2}+1+\frac{I C \cdot p}{1-p} .
$$

In the phase of data retrieval, if the desired data item is contained in the target index

bucket (with a probability of $\frac{B^{\prime}}{B(I-1)+B^{\prime}}$ ), we are done and no additional delay is incurred. Otherwise, the item is in a separate data bucket (with a probability of $\frac{B(I-1)}{B(I-1)+B^{\prime}}$ ); the average latency for retrieving the data bucket in an error-free environment is $\frac{I}{2}$, and we will have an additional delay of $i \cdot I C$ if the data bucket is corrupted continuously for $i$ times. The average access latency for this phase is:

$$
\begin{aligned}
E\left(d_{d}\right) & =0 \cdot \frac{B^{\prime}}{B(I-1)+B^{\prime}}+\left(\frac{I}{2}+\sum_{i=0}^{\infty} p^{i}(1-p) \cdot i \cdot I C\right) \cdot \frac{B(I-1)}{B(I-1)+B^{\prime}} \\
& =\left(\frac{I}{2}+\frac{I C \cdot p}{1-p}\right) \cdot \frac{B(I-1)}{B(I-1)+B^{\prime}}
\end{aligned}
$$

Therefore, the average overall access latency is obtained as follows:

$$
E(d)=E\left(d_{p i}\right)+E\left(d_{d}\right)
$$

where $E\left(t_{p i}\right)$ and $E\left(d_{d}\right)$ are given in (15) and (16), respectively. 
Next, we analyze the average tuning time. Suppose the client tunes into the broadcast right before the $j$ th $(1 \leq j \leq I)$ bucket of a chunk. The average tuning time until an error-free index bucket is received can be generally expressed by:

$$
\sum_{i=0}^{\infty} p^{i}(1-p) \cdot x_{i j}
$$

where $x_{i j}$ is the tuning time if $i$ sequential buckets are all corrupted. If the $(i+1)$ th bucket is an index bucket, we are finished with the initial probe; otherwise it is a data bucket, and we follow its pointer to access the next index bucket, which requires a tuning time of $\frac{1}{1-p}$ on average. Hence, we have

$$
x_{i j}= \begin{cases}i+1, & \text { if } i=1-j, I+1-j, 2 I+1-j, \cdots \\ i+1+\frac{1}{1-p}, & \text { otherwise. }\end{cases}
$$

Averaging over all $j$ 's $(1 \leq j \leq I)$, we get the average tuning time for the initial probe phase:

$$
E\left(t_{p}\right)=\sum_{i=0}^{\infty} p^{i}(1-p) \cdot(i+1)+\frac{I-1}{I} \cdot \sum_{i=0}^{\infty} p^{i}(1-p) \cdot \frac{1}{1-p}=\frac{2 I-1}{I(1-p)} .
$$

To analyze the average tuning time for the index search phase, first we derive the tuning time $t(l)$ up to the moment when the target index bucket that is $l$ chunks away is received. This can be computed recursively:

$$
t(l)= \begin{cases}0, & \text { if } l=0 \\ t(l-x) \cdot(1-p)+t(l-1) \cdot p+1, & \text { if } l>0\end{cases}
$$

where $x$ is the maximum value less than or equal to $l$ in the set of $\left\{1,2,\lfloor r+2\rfloor, \cdots,\left\lfloor\frac{r^{n_{c}-1}-1}{r-1}\right\rfloor+1\right\}$.

For $l>0$, it is possible that the received target index bucket is corrupted; in this case, a new round of search (with $C-1$ chunks away from the next target index bucket) is started. If the target index bucket is corrupted continuously for $i$ times, $i$ more rounds of searches are needed. Hence, the overall tuning time is:

$$
t(l)+\sum_{i=1}^{\infty} p^{i}(1-p) \cdot i \cdot t(C-1)=t(l)+\frac{t(C-1) \cdot p}{1-p} \quad(l>0)
$$

Thus, the average tuning time for index search is given by:

$$
E\left(t_{i}\right)=\frac{1}{C}\left(t(0)+\sum_{l=1}^{C-1}\left(t(l)+\frac{t(C-1) \cdot p}{1-p}\right)\right) .
$$


In the data retrieval phase, if the desired data item is contained in the target index bucket, no additional tuning time is incurred. Otherwise, the item is in a separate data bucket; an additional tuning time of $i+1$ buckets is needed if the data bucket is corrupted continuously for $i$ times. The average tuning time for this phase is obtained as follows:

$$
\begin{aligned}
E\left(t_{d}\right) & =0 \cdot \frac{B^{\prime}}{B(I-1)+B^{\prime}}+\left(\sum_{i=0}^{\infty} p^{i}(1-p) \cdot(i+1)\right) \cdot \frac{B(I-1)}{B(I-1)+B^{\prime}} \\
& =\frac{B(I-1)}{\left(B(I-1)+B^{\prime}\right) \cdot(1-p)} .
\end{aligned}
$$

The average overall tuning time is thus given by:

$$
E(t)=E\left(t_{p}\right)+E\left(t_{i}\right)+E\left(t_{d}\right),
$$

where $E\left(t_{p}\right), E\left(t_{i}\right)$, and $E\left(t_{d}\right)$ are obtained in (20), (23), and (24), respectively. 